\title{
Electromagnetic control based on Lie symmetry transformation
}

\author{
Zheng Mingliang \\ School of mechanical and electrical Engineering, Taihu University of Wuxi, Wuxi, 214064, China. \\ Corresponding Author: zhmlwxcstu@163.com
}

Submitted : 23/06/2020

Revised :08/08/2021

Accepted : 24/08/2021

\begin{abstract}
A design method of electromagnetic metamaterial based on Lie symmetry of Maxwell's equation is proposed, which is applied to the modulation of electromagnetic wave/light. Firstly, the electromagnetic control model based on metamaterials is introduced. Then, according to the theory of Transformation Optics (TO), Lie symmetry analysis is applied to the coordinate transformation of material physical space, and the key core is the determining equations of Lie symmetry derived. Secondly, the analytical forms of constitutive parameters (permittivity and permeability) of metamaterials are introduced, which can be used to design all kinds of electromagnetic metamaterials. Finally, the Lie symmetry method is applied to the control of electromagnetic beam width. The results show that the metamaterial based on Lie symmetry of Maxwell's equation has good field distribution, and it overcomes the single subjectivity of traditional coordinate transformation in optical transformation. The wave simulation by COMSOL Multiphysics software verifies the correctness of Lie symmetry method.
\end{abstract}

Key words: Lie symmetry; metamaterials; Constitutive parameters; Electromagnetic control.

\section{INTRODUCTION}

In order to control the propagation of electromagnetic wave flexibly and effectively, people have been looking for some artificial materials to replace conventional materials, and the metamaterials are a kind of artificial electromagnetic functional materials that can achieve specific permittivity and permeability (T. Li et al., 2009). The basic principle of electromagnetic field control by metamaterials is optical transformation. Its core is to find out the mapping function of coordinate transformation and then derive the tensors of medium constitutive parameters. At present, we can control the electromagnetic wave path (such as designing the invisibility cloaks (J. B. Pendry et al., 2006) and beam deflectors (M. Rahm et al., 2008) and the amplitude of electromagnetic field (such as designing the field concentrators with gathering electromagnetic wave energy (M. Rahm et al., 2008)). In recent years, we can also use the metamaterials to control the phase (polarization) of electromagnetic wave, such as designing the polarization rotators (D. H. Kwon et al., 2008) and the rotating media layer of field phase (Y. H. Chen et al., 2007). The latest literature on the preparation properties and applications of artificial electromagnetic metamaterials is shown by Q. Q. Zhang et al., 2021; S. Hu et al., 2020; M. Karthik et al., 2019; X. F. Zhu et al., 2019; Litchinitser, 2018; K. Thomas et al., 2017, and so on. 
Transformation Optics (D. Schurig et al., 2006) is a process of transforming geometric space, which belongs to variable substitution in mathematics. However, the existing coordinate transformations in the design of metamaterials have always been based on experience or experiment, and there is not a unified method to provide sufficient design scheme of material constitutive parameters. So, at present, there have been some difficulties concerning the realization of electromagnetic control devices by traditional coordinate transformation. Lie symmetry analysis (N. H. Ibragimov et al., 2013) is a powerful tool to study the invariance of differential equations. It can obtain the coordinate forms of group transformation systematically and comprehensively. In this paper, we try to use Lie symmetry transformation to solve the invariance of Maxwell's equation and try to get enough mapping functions of coordinate transformation, so as to select the optimal constitutive parameter of materials and realize the propagation of electromagnetic field that follows the expected trajectory.

\section{ELECTROMAGNETIC CONTROL BASED ON METAMATERIAL}

The propagation path of electromagnetic wave is shown in Figure 1. The outside electromagnetic wave is incident from the left side. Without using metamaterials, the medium of area 1, area 2, and area 3 is all general air. The protection area is in area 3, with the increase of propagation distance. Figure 1(b) shows that the electromagnetic wave will scatter at the protection area. So, it will cause electromagnetic radiation, decrease of optical power density, and instability of beam width.
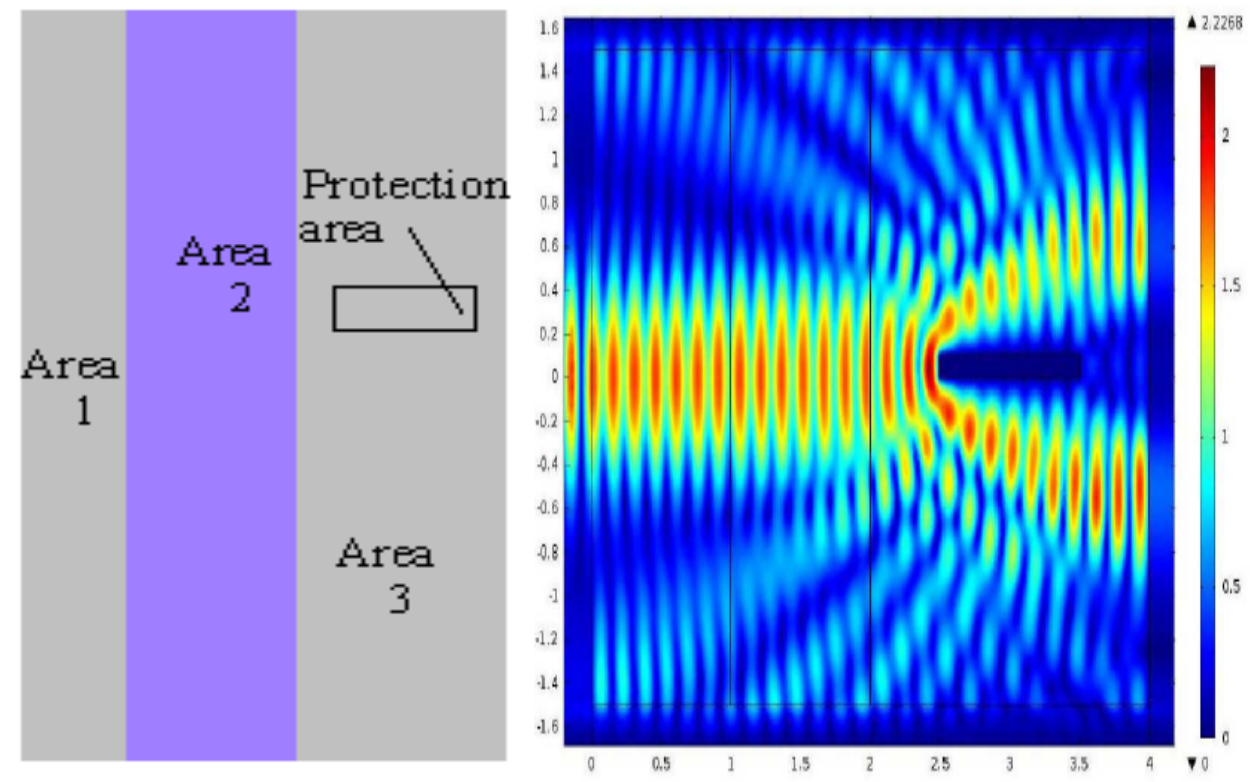

(a) Propagation area of electromagnetic wave. (b) Scattering effect of electromagnetic wave.

Figure 1. Propagation and radiation of electromagnetic wave.

Electromagnetic control includes the propagation direction, amplitude, phase, and beam width of electromagnetic wave. Especially, the modulation of Gaussian beam width is a very important subject. The principle of beam width modulation based on metamaterials (C. Li, et al., 2012) is to design the permittivity and permeability of medium by using a certain form of coordinate transformation, so as to obtain a specific distribution of refractive index. Eventually, it can compress and broaden the beam width and collimate the beam without 
divergence. The method is to introduce anisotropic metamaterials into area 2 to realize the convergence or focusing of beam. So, the beam will maintain a great energy density and continue to forward propagation. This change is to design the material characteristics for the actual physical propagation path of wave. Figure 2 shows the special unit structure of artificial composite metamaterials, which has a ring-shaped structure on the front and a resonance rod centered on the back. Figure 3 shows that a specific metamaterial medium in area 2 can transform a Gaussian beam with a certain waist width into an approximate linear beam, and the center of the linear beam has a great energy density, which will continue to travel, and the beam propagation on both sides of the flat medium is nonreflective.

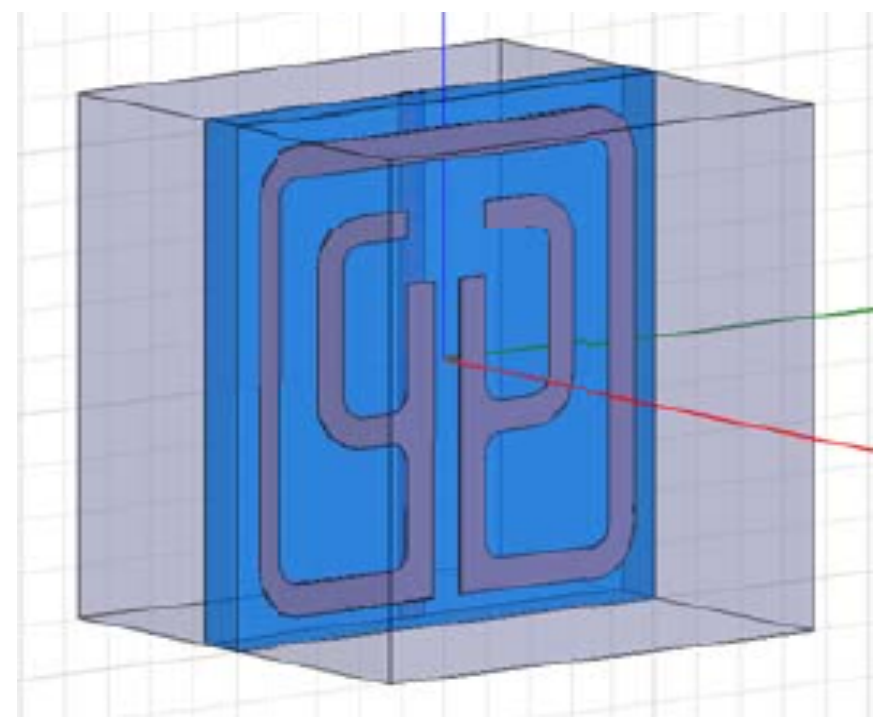

Figure 2. Unit structure model of electromagnetic metamaterial.

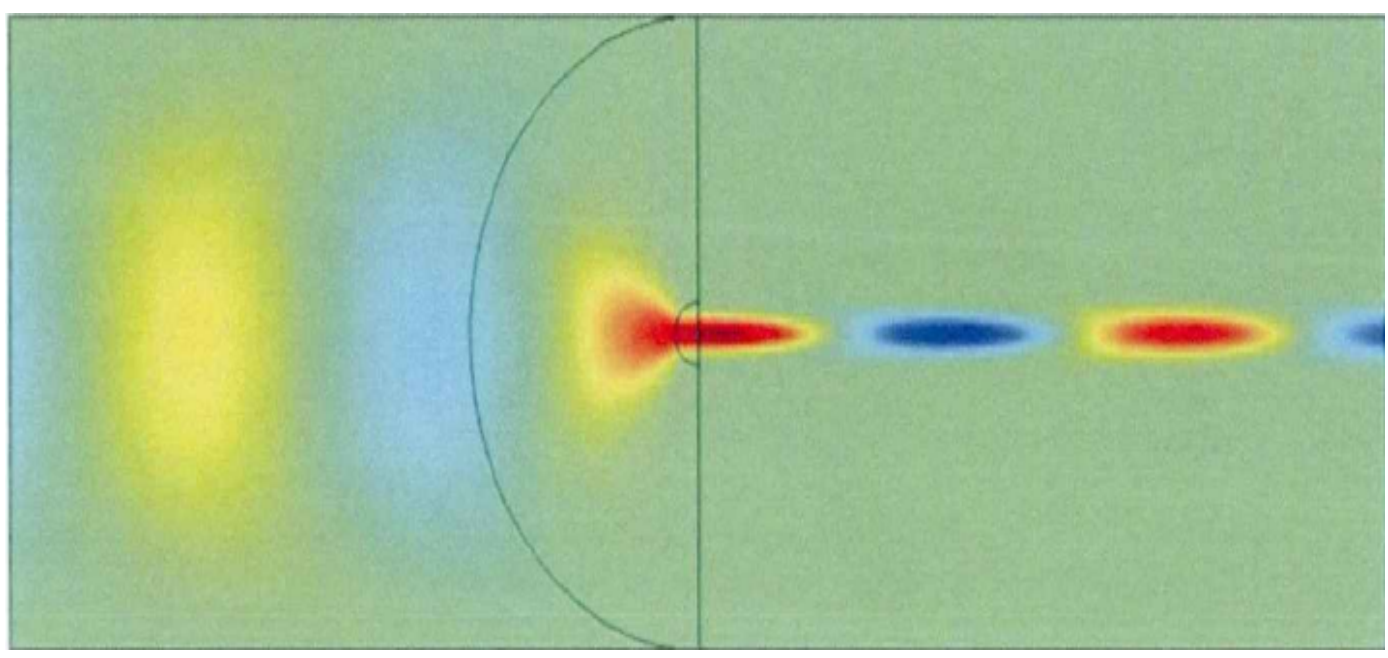

Figure 3. The convergence or focusing of beam. 


\section{LIE SYMMETRY TRANSFORMATION AND MATERIAL CONSTITUTIVE PARAMETERS}

Maxwell's equation is the basic equation of electromagnetic field, which expresses the basic law of macroscopic electromagnetic phenomenon. Its expression is as follows (Z. W. Qin et al., 2014):

$$
\begin{aligned}
& \nabla \times \mathbf{H}(\mathbf{r}, t)=\frac{\partial \mathbf{D}(\mathbf{r}, t)}{\partial t}+\mathbf{J}(\mathbf{r}, t), \\
& \nabla \times \mathbf{E}(\mathbf{r}, t)=-\frac{\partial \mathbf{B}(\mathbf{r}, t)}{\partial t}, \\
& \nabla \cdot \mathbf{D}(\mathbf{r}, t)=\rho(\mathbf{r}, t), \\
& \nabla \cdot \mathbf{B}(\mathbf{r}, t)=0, \\
& \mathbf{D}=\varepsilon \cdot \mathbf{E}, \mathbf{B}=\mu \cdot \mathbf{H}, \mathbf{J}=\sigma \cdot \mathbf{E}
\end{aligned}
$$

Here, $\nabla=\frac{\partial}{\partial x} \vec{i}+\frac{\partial}{\partial y} \vec{j}+\frac{\partial}{\partial k} \vec{k}$, and $\mathbf{E}, \mathbf{B}, \mathbf{H}, \mathbf{D}, \mathbf{J}, \rho$ are the electric intensity, magnetic flux density, magnetic density, electric potential, ampere density, and charge density. The constitutive parameters $\varepsilon, \mu, \sigma$ are permittivity, permeability, and conductivity, which are expressed in tensor form.

Considering the Cartesian coordinate space for the inhomogeneous medium in passive region, Equation (1) can be further simplified to (A. J. Ward et al., 1996)

$$
\begin{aligned}
& \nabla \times \mathbf{H}(x, y, z, t)=\varepsilon_{r} \varepsilon_{0} \frac{\partial \mathbf{E}}{\partial t}, \\
& \nabla \times \mathbf{E}(x, y, z, t)=-\mu_{r} \mu_{0} \frac{\partial \mathbf{H}}{\partial t}
\end{aligned}
$$

$\varepsilon=\varepsilon_{r} \varepsilon_{0}, \mu=\mu_{r} \mu_{0}, \varepsilon_{0}, \mu_{0}$ are constants.

The independent variables are $\mathbf{x}=(t, x, y, z)^{T}$, the dependent variables are

$$
\mathbf{u}=\left(E_{1}, E_{2}, E_{3}, H_{1}, H_{2}, H_{3}\right)^{T} \text {, and the differential operator is } D \text {. The first and second partial }
$$
derivatives are

$$
\begin{aligned}
& u_{\mathrm{i}}^{\alpha}=D_{\mathrm{i}}\left(u^{\alpha}\right), u_{\mathrm{ij}}^{\alpha}=D_{\mathrm{i}}\left(u_{\mathrm{j}}^{\alpha}\right)=D_{\mathrm{i}} D_{\mathrm{j}}\left(u^{\alpha}\right) \\
& (\alpha=1,2,3,4,5,6 ; i, j=1,2,3,4)
\end{aligned}
$$

Considering a transformation group with a single parameter: 


$$
\begin{aligned}
& \bar{t}=t+\delta \xi_{1}(\mathbf{x}, \mathbf{u}), \bar{x}=x+\delta \xi_{2}(\mathbf{x}, \mathbf{u}), \bar{y}=y+\delta \xi_{3}(\mathbf{x}, \mathbf{u}), \bar{z}=z+\delta \xi_{4}(\mathbf{x}, \mathbf{u}) \\
& \bar{H}_{1}=H_{1}+\delta \kappa_{1}(\mathbf{x}, \mathbf{u}), \bar{H}_{2}=H_{2}+\delta \kappa_{2}(\mathbf{x}, \mathbf{u}), \bar{H}_{3}=H_{3}+\delta \kappa_{3}(\mathbf{x}, \mathbf{u}), \\
& \bar{E}_{1}=E_{1}+\delta \kappa_{4}(\mathbf{x}, \mathbf{u}), \bar{E}_{2}=E_{2}+\delta \kappa_{5}(\mathbf{x}, \mathbf{u}), \bar{E}_{3}=E_{3}+\delta \kappa_{6}(\mathbf{x}, \mathbf{u}) .
\end{aligned}
$$

Here $\delta$ is an infinitesimal parameter, and $\xi_{i}, \kappa_{w}$ are generator functions.

According to the extension theory of Lie group, the generator, the first- and second-order vectors of transformation (4) are as follows:

$$
\begin{aligned}
& X=\xi_{i}(\mathbf{x}, \mathbf{u}) \frac{\partial}{\partial x_{i}}+\kappa_{r}(\mathbf{x}, \mathbf{u}) \frac{\partial}{\partial u^{r}}, X^{(1)}=X+\zeta_{i}^{(1) w}(\mathbf{x}, \mathbf{u}, \partial \mathbf{u}) \frac{\partial}{\partial u_{i}^{w}} \\
& \zeta_{i}^{(1) w}=D_{i} \kappa^{w}-\left(D_{i} \xi_{j}\right) u_{j}^{w},(i, j=1,2,3,4 ; r, w=1,2,3,4,5,6)
\end{aligned}
$$

The invariance of Equations (2) under transformation (4) can be expressed as follows:

$$
\begin{aligned}
& X^{(1)}\left[\nabla \times \mathbf{H}(\mathbf{r}, t)-\varepsilon_{r} \varepsilon_{0} \frac{\partial \mathbf{E}}{\partial t}\right]=0, \\
& X^{(1)}\left[\nabla \times \mathbf{E}(\mathbf{r}, t)+\mu_{r} \mu_{0} \frac{\partial \mathbf{H}}{\partial t}\right]=0 .
\end{aligned}
$$

Equations (6) are the determining equations of Lie symmetry. By expanding the first-order partial differential Equations (6), the solutions of $\xi_{i}, \kappa_{w}$ can be obtained.

The permittivity and permeability of the transformed material medium are as follows:

$$
\begin{aligned}
& \bar{\varepsilon}_{r}(\bar{x}, \bar{y}, \bar{z},)=\varepsilon_{r}\left(\bar{x}-\delta \xi_{2}, \bar{y}-\delta \xi_{3}, \bar{z}-\delta \xi_{4}\right) \\
& \bar{\mu}_{r}(\bar{x}, \bar{y}, \bar{z})=\mu_{r}\left(\bar{x}-\delta \xi_{2}, \bar{y}-\delta \xi_{3}, \bar{z}-\delta \xi_{4}\right)
\end{aligned}
$$

Equation (7) shows that when the same materials are placed in different spaces, the tensors of material property will be different due to the space geometry topology.

\section{ANALYSIS ON APPLICATION}

As shown in Figure 4, the beam width of the electromagnetic wave is compressed or expanded by adding a metamaterial medium plate with the thickness $d$ (it is greater than the wavelength of beam). 

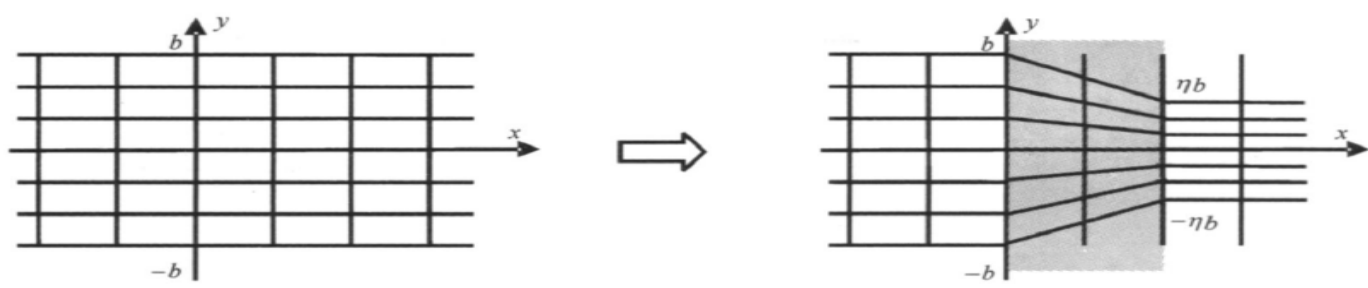

(a) original system
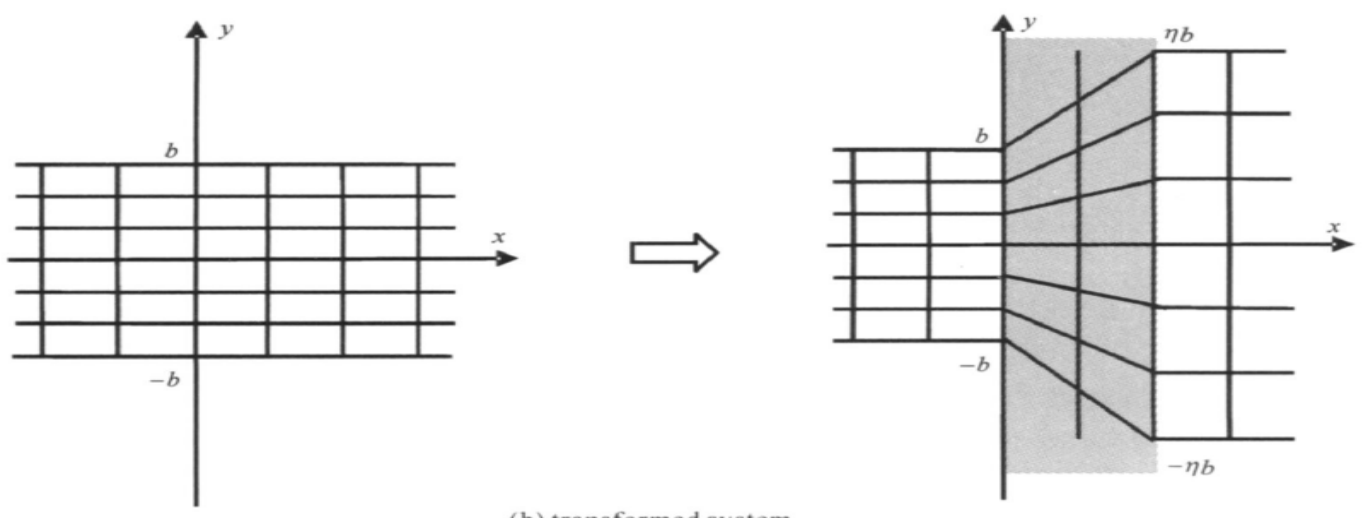

(b) transformed system

Figure 4. Beam width modulation of electromagnetic wave.

The original area is free space with air medium. So the tensors of permittivity and permeability are

$$
\varepsilon_{r}^{i j}=\mu_{r}^{i j}=\left[\begin{array}{lll}
1 & 0 & 0 \\
0 & 1 & 0 \\
0 & 0 & 1
\end{array}\right]
$$

Setting $\delta=\frac{1}{d}$, we take the spatial migration transformation for (6). That is to say $\xi_{i}, \kappa_{w}$ are

$$
\begin{aligned}
& \xi_{1}=0, \xi_{2}=0, \xi_{3}=(\eta-1) x y, \xi_{4}=0, \\
& \kappa_{1}=0, \kappa_{2}=0, \kappa_{3}=0, \kappa_{4}=0, \kappa_{5}=0, \kappa_{6}=0 .
\end{aligned}
$$

Here, $\eta$ is an arbitrary constant, which is also known as the adjustment coefficient for electromagnetic control, and it can change the form of coordinate transformation for metamaterial.

Then, the corresponding coordinate transformation is

$$
\bar{t}=t, \bar{x}=x, \bar{y}=y+\frac{1}{d}(\eta-1) x y, \bar{z}=z .
$$



follows:

According to Equations (7), the relative permittivity and permeability of the metamaterial medium plate are as

$$
\bar{\varepsilon}_{r}^{i j}=\bar{\mu}_{r}^{i j}=\left[\begin{array}{ccc}
1 & (\eta-1) y / d & 0 \\
(\eta-1) y / d & {[(\eta-1) y / d]^{2}+[1+(\eta-1) x / d]^{2}} & 0 \\
0 & 0 & 1
\end{array}\right]
$$

Equation (11) shows that the beam width path guidance can be adjusted by setting different $\eta$ when plate with the thickness $d$ is stationary.

The center coordinate $(x, y)$ of metamaterial medium plate in plane area is $(d / 2,0)$, in center point. So, the determinant of tensor for permittivity or permeability is

$$
\operatorname{det}\left(\bar{\varepsilon}_{r}^{i j}\right)=\operatorname{det}\left(\bar{\mu}_{r}^{i j}\right)=(\eta+1)^{2} / 4
$$

In center point of metamaterial medium plate, the curve that shows that the determinant of tensor permittivity varies with $\eta$ is shown in Figure 5, showing that the determinant decreases first and then increases, and there is a zero extreme point when $\eta=-1$.

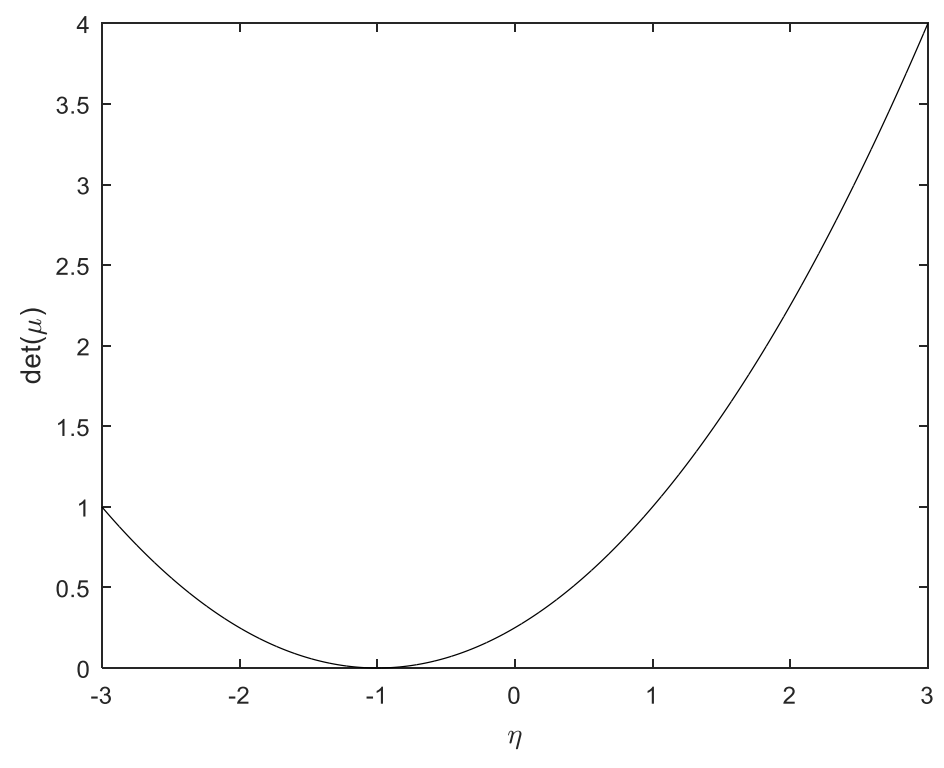

Figure 5. The determinant of tensor for permeability varies with $\eta$.

In order to verify the electromagnetic properties of the metamaterial, we use the finite element method to fullwave simulation (COMSOL). Considering a Gaussian plane wave with wave length, $\lambda_{0}=1.5 \mu \mathrm{m}$ in transverse magnetic field (R. L. Wang et al., 2011). The width of plate is $d=3 \lambda_{0}$, which is the optimum wave width ratio for the beam to continue to move forward without deformation and divergence (X. F. Lv, 2010), and the length of plate is $25 \lambda_{0}$. The adjustment coefficient for the generator of Lie symmetry is $\eta=0.5$, and the electromagnetic performance simulation is shown in Figure 6, which shows that the beam width becomes a half of previous level 
through the medium. So, the metamaterial plays the role of compressing electromagnetic wave. The adjustment coefficient $\eta=1.5$, and the simulation result is shown in Figure 7, which shows that the beam width is 1.5 times the previous level through the medium. So, the metamaterial plays the role of broadening electromagnetic wave. Therefore, the software simulation is consistent with the theoretical calculation result.
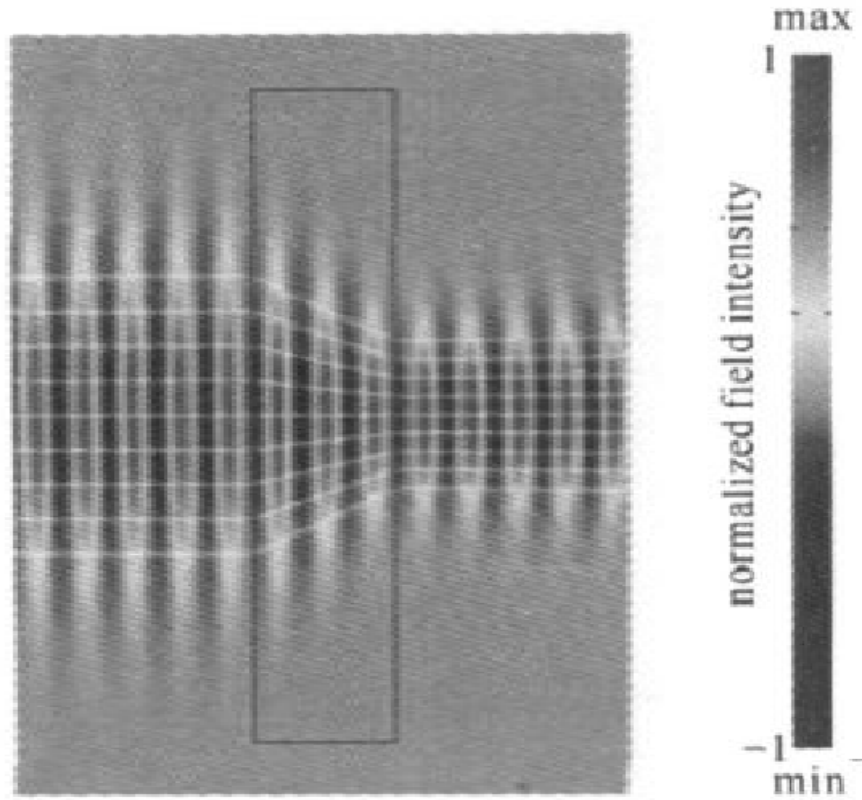

Figure 6. Electromagnetic wave modulation of $\eta=0.5$.
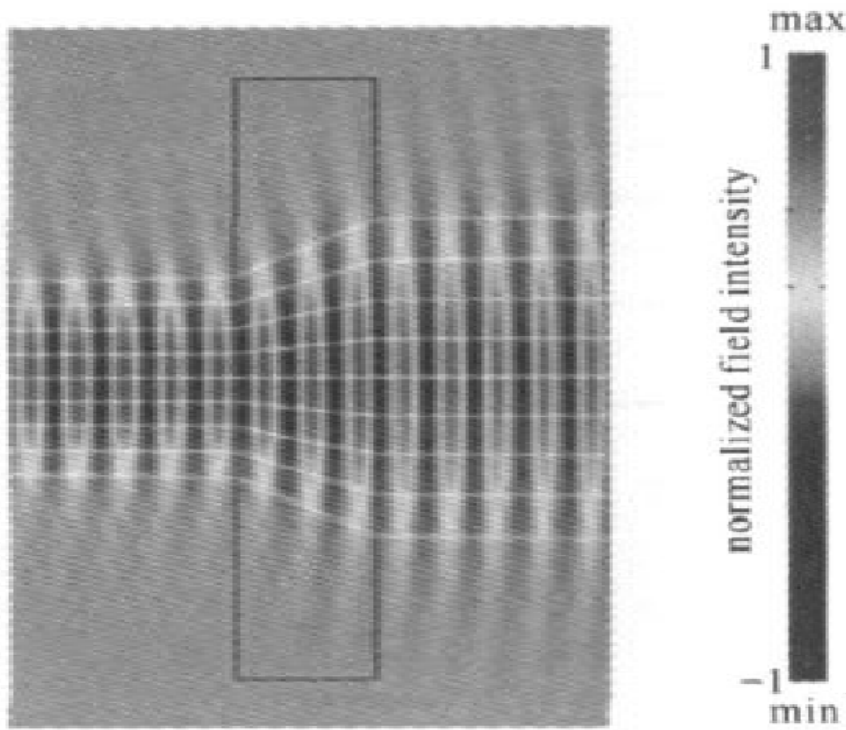

Figure 7. Electromagnetic wave modulation of $\eta=1.5$. 


\section{CONCLUSION}

We use Lie symmetry of Maxwell's equation to obtain the supernormal material medium, which can compress or expand the beam width of the incident Gaussian beam, and even compress a Gaussian beam with a certain beam width into a line beam with a very thin beam width. This method is not limited by the intensity of electromagnetic propagation. Its basic principle is using Lie symmetry to solve Maxwell's equation in order to obtain the analytical tensor forms of permittivity and permeability of metamaterials. Its key is to solve the Lie symmetric generator functions, which determine the diversity of constitutive parameters of metamaterials. Meanwhile, the properties of metamaterials which can adjust the beam width of electromagnetic wave are calculated, and the influence of different Lie symmetry transformations on the path propagation is analyzed. The full-wave simulation is carried out using the COMSOL Multiphysics software, and the correctness of theory is verified. The results can be used for reference in the design of various structures of artificial electromagnetic metamaterials or optical elements.

\section{REFERENCES}

Li, T., Yang, J., Huang, M., et al. 2011. Arbitrary control of the electromagnetic field in two-dimensional waveguide and slit using metamaterials. Applied Physics A 105 (2): 509-515.

Pendry, J. B., Schurig, D., Smith, D. R. 2006. Controlling electromagnetic fields. Science 312: 1780-1782.

Rahm, M., Cummer, S. A., Schurig, D., et al. 2008. Optical design of refectionless complex media by finite embedded coordinate transformations. Phys. Rev. Lett. 100: 063903.

Rahm, M., Schurig, D., Roberts, D. A., et al. 2008. Design of electromagnetic cloaks and concentrators using form-invariant coordinate transformations of Maxwell's equations. Photon NanostructFundamApplic 6(1): $87-95$.

Kwon, D. H., Werner, D. H. 2008. Polarization sputter and polarization rotator designs based on transformationoptics. Opt. Express 16(23): 18731-18738.

Chen, H, Y, Chan, C. T. 2007. Transformation media that rotate electromagnetic fields. Appl. Phys. Lett. 90: 241105.

Schurig, D., Pendry, J. B., Smith, D. R. 2006. Calculation of material properties and ray tracing in transformation media. Opt. Express 14(21): 9794-9804.

Ibragimov, N. H. 2013. Differential equations and problems of mathematical physics. Beijing: Higher Education Press. 101-123. (in Chinese)

Li, C., Li, J. B., Xin. P. 2012. Research on electromagnetic shielding based on optical transformation. Science and Technology Communication 210(2): 132-133. (in Chinese)

Qin, Z. W. 2014. A structure design research of electromagnetic stealth based on the form invariance principle of Maxwell's epuation. Hangzhou: Zhejiang University. 10-12. (in Chinese)

Ward, A. J., Pendry, J. B. 1996. Refraction and geometry in Maxwell's equations. Journal of Modern Optics 43(4): 773-793.

Wang, R. L., Mo, L. Z., Zhang, J. 2011. Modulation of electromagnetic beamwidth based on transformation optics. High Power Laser and Particle Beams 23(3): 633-637. (in Chinese).

Lv, X. F. 2010. Research of beam manipulation using metamaterials. Changsha: Hunan University. 30-35. (in Chinese)

Zhang, Q. Q., Zhu, W.,Feng, J. J., et al. 2021. High-efficient heat flux manipulation of micro-scale thermal metamaterials with facile functional unit design. Materials \& Design204: 109657. 
Hu, S., Liu, D., Yang, H. L. 2020. Modulation of electromagnetic beamwidth based on transformation opticsManipulation of electromagnetically induced transparency in all-dielectric metamaterials: From onoff to double transparent windows. Optik 223: 165637.

Karthik M., James M. M. 2019. Extraordinary wave manipulation characteristics of nonlinear inertant acoustic metamaterials. Journal of the Franklin Institute 356 (14): 7731-7753.

ZhuX. F., LauS. K. 2019. Reflected wave manipulation via acoustic metamaterials with decoupled amplitude and phase. Applied Physics A 125 (6):1-7.

Litchinitser. 2018. Nonlinear optics in metamaterials. Advances in Physics: X 3 (1): 1367628.

Thomas K., Costas M. S., Martin W. 2017. Metamaterials in microwaves, optics, mechanics, thermodynamics, and transport. Journal of Optics 19 (8): 084005. 